\title{
Sensitivity enhancement for measurement of nonlinear refraction using top-hat beams
}

\author{
Junyi Yang $\cdot$ Xingzhi Wu $\cdot$ Min Shi $\cdot$ \\ Zhongguo Li $\cdot$ Yong Yang $\cdot$ Yinglin Song
}

Received: 24 September 2012/ Accepted: 11 December 2012/Published online: 13 January 2013

(C) The Author(s) 2013. This article is published with open access at Springerlink.com

\begin{abstract}
A simple and high-sensitivity technique is presented to investigate nonlinear refraction. In this technique, a combination of aperture and obscuration disk is introduced in the measurement system. Compared to the top-hat Z-scan, the curves of modified top-hat Z-scan for the nonlinear refraction show a single peak. Furthermore, the sensitivity of this new technique can be more than two orders of magnitude enhanced. Nonlinear refraction of Nddoped phosphate glass is investigated using this technique with 19 ps pulses at wavelength of $532 \mathrm{~nm}$.
\end{abstract}

\section{Introduction}

The Z-scan method was first provided by Sheik-Bahae et al. $[1,2]$ and has been employed extensively because of its simplicity, accuracy, and the ease of separation of nonlinear refraction (NLR) and nonlinear absorption (NLA). Since then, some alterative or improved Z-scan techniques have been developed such as Gaussian-Bessel Z-scan [3], top-hat Z-scan [4, 5], EZ-scan [6] and NER $Z$-scan [7]. The improvements of these Z-scan techniques are a large enhancement of sensitivity or extended application. Recently, several modified Z-scan techniques,

J. Yang $\cdot$ X. Wu $\cdot$ M. Shi $\cdot$ Y. Yang $\cdot$ Y. Song

School of Physical Science and Technology, Soochow

University, Suzhou 215006, People's Republic of China

J. Yang

Eoplly New Energy Technology Co. Ltd,

Nantong 226612, People's Republic of China

Z. Li · Y. Song $(\bowtie)$

Department of Physics, Harbin Institute of Technology,

Harbin 150001, People's Republic of China

e-mail: ylsong@hit.edu.cn which own higher sensitivity, have been presented [8,9]. The top-hat beam Z-scan [4] is extremely important in the family of Z-scan techniques. It not only exhibits the enhancement of sensitivity compared with the Gaussian beam Z-scan by a factor of 2.5 , but also the required spatial profile of the beam used is very easily produced. The sensitivity enhancement of EZ-scan can be an order-ofmagnitude compared to the Z-scan. However, this technique demands perfect spatial profile of the beam. A position sensitive detector (PSD) is used in the baryscan technique [7], in which nonlinear refraction is investigated by monitoring the barycentric position of a truncated laser beam as the sample is moved. At the same time, the spatial stability of laser beam and the resolution of the PSD have a great influence on the sensitivity of this technique. In addition, these techniques described above need the sample moving through the focal plane and multiple measurements under a strong laser pulse excitation. This can lead, in some cases, to the damage of the sample. The application of single-shot techniques would allow for overcoming the above difficulties such as $4 f$ coherent imaging system with a phase object (NIT-PO) [10].

As described in paper [6], the sensitivity enhancement of the EZ-scan is due to the larger fractional change in intensity in the wings of the beam compared to that near the center. However, the measurement accuracy of this method is very dependent on the perfect profile of the Gaussian beam which is difficult to acquire. Top-hat beam can be readily obtained by taking a small portion of the expanded beams from lasers with arbitrary beam profiles. In this paper, a technique using top-hat beam with a combination of aperture and obscuration disk is presented. The sensitivity of measurement is greatly enhanced, and a single-shot technique based on this technique for measurement of nonlinear refraction is also described briefly. 


\section{Theoretical model and discussion}

In the far field, the change of the spatial distribution of the intensity for top-hat beam is very different from that for Gaussian beam when the nonlinear phase shift exists. For Gaussian beam, the intensity of the wings is reduced smoothly according to the Gaussian function in initial condition. However, the intensity of the wings for top-hat beam in the far field is very small at first, and the intensity change is increased with the nonlinear phase shift increased, as shown in Fig. 3 in the paper [11]. So it can be expected that the curves of top-hat Z-scan with an obscuration disk for the nonlinear refraction show a single peak, and reach maximum when the sample is placed at the focus. Furthermore, as described in EZ-scan technique, the change in intensity in the wings of the top-hat beam is very larger than that near the center of beam, so the sensitivity is greatly enhanced.

Initially, only an obscuration disk is used in the modified top-hat Z-scan technique. It is found that the sensitivity is enhanced only several times compared to that of the top-hat Z-scan. The sensitivity is not as large as expected, which is attributed to the background noise. An aperture is added in the front of the disk in order to block some noise. Thus, the field in front of the disk is also modulated. The normalized transmittance changes greatly with the size of the aperture. Figure 1 shows the normalized transmittance of the modified top-hat Z-scan with the size of the aperture theoretically while the sample is placed at the focal plane of the lens. We think that the aperture with appropriate size can optimize the coherence of the modulation light (induced by the nonlinear phase shift) with the initial intensity of the wings, so the sensitivity is greatly enhanced.

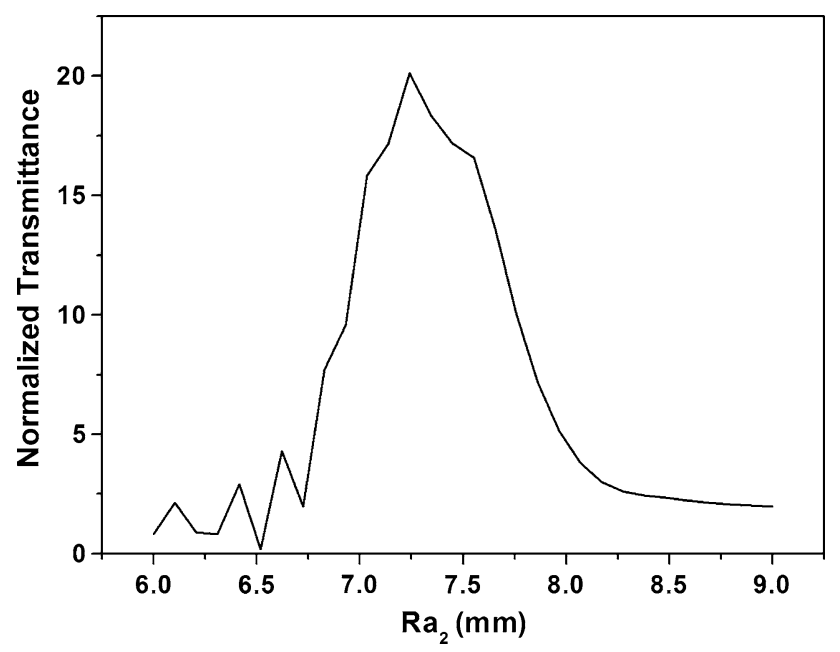

Fig. 1 The normalized transmittance as a function of the radius of the aperture $A_{2}$
Figure 2 shows the modified top-hat beam Z-scan arrangement. The laser beam was expanded and subsequently illuminated a circular aperture $\mathrm{A}_{1}$ with a diameter $d$ to generate a top-hat beam, and then was focused by a lens $\mathrm{L}_{1}$ with a focal length of $f$. The transmitted pulse energy in the presence of the far field disk was probed by a detector $\mathrm{D}_{2}$, as a function of sample position $z$. The branch spitted by the beam splitter BS is detected by the detector $\mathrm{D}_{1}$ to record the energy of input laser pulse.

The experimental Z-scan traces using the top-hat beam with the combination of disk and aperture in the far field for $\mathrm{CS}_{2}$ is shown in Fig. 3. The energy of the input laser pulse is $90 \mathrm{~nJ}$ and the main parameters used in our numerical simulation are same with our experimental conditions which we will describe below. Obviously, the trace of top-hat Z-scan with the combination of disk and aperture shows a single peak shape, which is very different from the general Z-scan (Z-scan, top-hat Z-scan and EZscan) trace. The sensitivity of the modified top-hat Z-scan is larger than two order-of-magnitudes comparing with the conventional top-hat Z-scan technique. The peak-to-valley normalized transmittance $\Delta T_{p-v}$ of the top-hat Z-scan is about 0.1 .

It is found that when the distance between the aperture $\mathrm{A}_{1}$, the convex lens $\mathrm{L}_{3}$ and the disk satisfies the relationship of geometrical optics imaging of the lens, the lens $L_{1}$ will image the space shape of the aperture $A_{1}$ to the disk. Then the disk blocks most of the low spatial frequency light, and the high spatial frequency is passed, which leads to the sensitivity enhanced several times, as shown in Fig. 3. Theoretically, the aperture should not be used here, because it decreases the sensitivity of the measurement. However, it must be used under our experimental conditions to block the noise and must be considered in the theoretical fitting.

On the plane $z$ the transverse field distribution of a focused top-hat beam can be described, according to the Fresnel diffraction theory of propagation of electromagnetic wave, as follows:

$E(r, z, t) \infty \exp \left[-{\frac{t}{2 \tau^{2}}}^{2}\right] \frac{2 J_{1}\left(\pi r / \omega_{0}\right)}{\pi r / \omega_{0}} \otimes \frac{\exp \left(i \pi r^{2} / \lambda z\right)}{\lambda z}$

where $r$ is the transverse polar coordinate, $\lambda$ is the wavelength of the laser beam, $J_{1}()$ is the first-order Bessel function. The waist of the focused top-hat beam and the Rayleigh range are defined with the same rule as in [4]; i.e., $\omega_{0}=\lambda f / d, z_{0}=\pi \omega_{0}^{2} / \lambda$ is the diffraction length of the beam, $\tau$ is the laser pulse width.

For simplification, only the third-order nonlinear refraction is investigated. Considering a thin medium and using the slowly varying envelope approximation, in the sample, the beam propagating equations for the phase is 
Fig. 2 Schematic of the modified top-hat beam Z-scan experiment: $A_{1}, A_{2}$ apertures, $L$ lens with focal length $f$,

$D$ detector

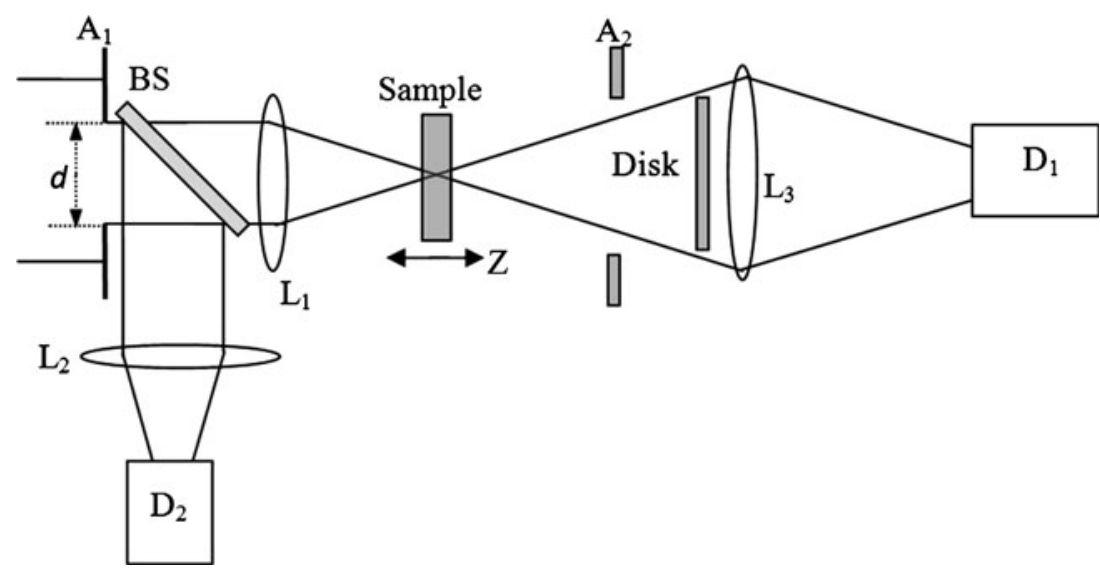

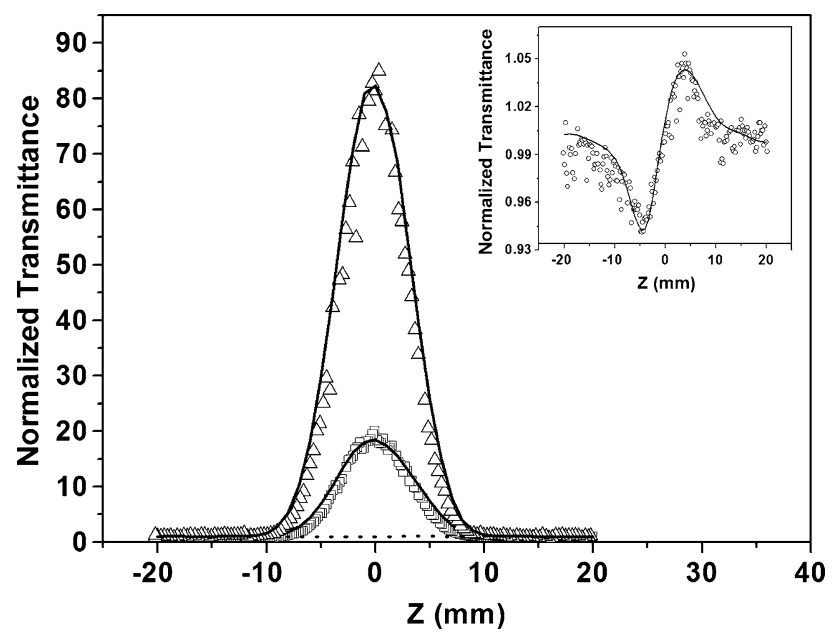

Fig. 3 The experimental traces for $\mathrm{CS}_{2}$ of top-hat Z-scan with a combination of aperture and disk (the distance between the aperture $A_{1}$, the convex lens $L_{3}$ and the disk does not satisfy the relationship of geometrical optics imaging of lens (square), the distance satisfies the relationship of geometrical optics imaging of lens (triangle), and an aperture (tripple dotted) in the far field). The solid line is the theoretical fit to the experimental result. The inset is the experimental traces of top-hat $\mathrm{Z}$-scan for $\mathrm{CS}_{2}$ under the same experimental conditions

$\frac{d \Delta \phi}{d z^{\prime}}=k \gamma I$

Here, $\Delta \phi$ represent the phase change, $z^{\prime}$ is the propagation length in sample, $\gamma$ is the nonlinear refraction coefficients, and $I$ is the intensity in the sample, $I=\left|E_{0}\right|^{2}$ at $z^{\prime}=0$.

The amplitude of the field in rear surface of the sample can be written as:

$E_{01}\left(r_{1}, z, t\right)=E_{0}\left(r_{1}, z, t\right) \exp (-\alpha L) \exp \left[i \Delta \phi\left(r_{1}, t\right)\right]$

The amplitude of the field at the aperture and disk can be achieved with two Fresnel diffraction integrals. The field of the disk is defined as $E_{d}\left(r_{2}, z, t\right)$.

Similarly, in the linear case, the field of the aperture and disk can also be obtained, and the field of the disk defined as $E_{\mathrm{d}}^{\prime}\left(r_{2}, z, t\right)$. The integration of irradiance over the pulse duration and the cross-section of the periphery of the disk give an energy corresponding to that detected by $\mathrm{D}_{2}$. Then the normalized nonlinear transmittance $T$ can be calculated as:

$T(z)=\frac{\int_{-\infty}^{+\infty} \int_{r_{d}}^{+\infty} 2 \pi r_{2}\left|E_{d}\right|^{2} d r_{2} d t}{\int_{-\infty}^{+\infty} \int_{r_{d}}^{+\infty} 2 \pi r_{2}\left|E_{d}^{\prime}\right|^{2} d r_{2} d t}$

The nonlinear refraction coefficient $\gamma$ can be deduced by fitting the numerical calculated nonlinear transmittance to the experimental nonlinear transmittance.

More important, as shown in Fig. 3, it is different from the valley-peak shape for the positive in the top-hat Z-scan and EZ-scan measurement, only single peak shape for nonlinear refraction is observed in the modified top-hat Z-scan, which means that the nonlinear refraction index can be obtained using a single-shot pulse, in which the sample is placed in position and no displacement. It means that this modified technique can be easily extended to be other techniques to investigate optical nonlinearities due to the single-shot pulse measurement, such as the timeresolved pump-probe with high sensitivity.

\section{Experiment}

The Nd-doped phosphate glass sample (SIOM NAP2, $\mathrm{Nd} 2 \mathrm{O} 30.5 \mathrm{wt} \%$ ) has been used as the sample in our experiment. The preparation of this glass is similar to Ref. [12]. Nd-doped phosphate laser glass is widely used in high-peak power solid state laser systems. And such laser glass must also have low optical nonlinearity, thus avoiding damage due to the presence of self-focusing of the glass. A mode-locked Nd:YAG laser (EKSPLA, PL2143B) with a pulse width of 19 ps (FWHM), repetition rate of $2 \mathrm{~Hz}$, at wavelength of $532 \mathrm{~nm}$ is used to excite the sample. The energy of the input laser pulse is $1.3 \mu \mathrm{J}$. The diameter of the circular aperture $\mathrm{A}_{1}$ is $3.4 \mathrm{~mm}$. The focal length of $L$ is 


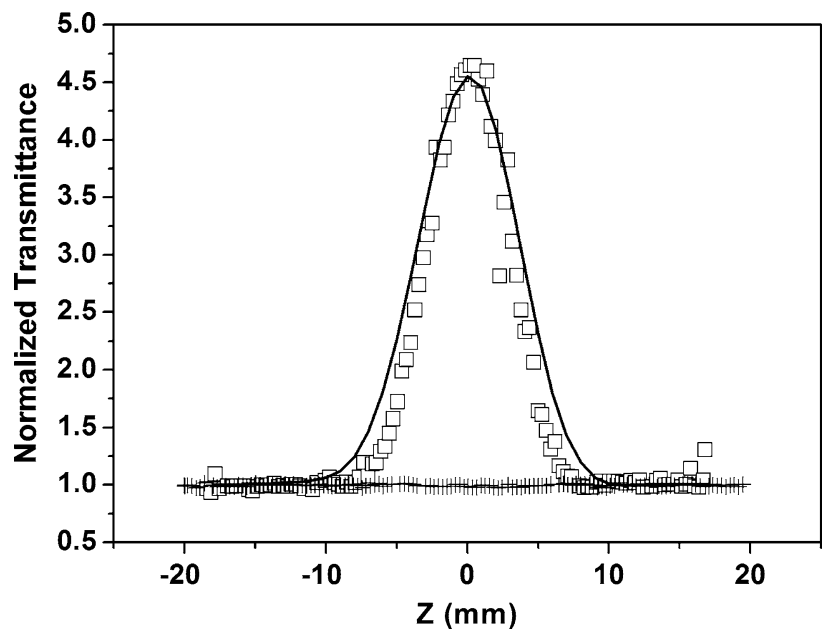

Fig. 4 The experimental traces for NAP2 of top-hat Z-scan (plus) and the modified Z-scan with a combination of aperture and disk (square)

$412 \mathrm{~mm}$ giving a beam waist $\omega_{0}=33 \mu \mathrm{m}$ at the focus. The thickness of the glass is $1 \mathrm{~mm}$. The transmittance of the sample is measured with a disk in the far field. The diameter of aperture $\mathrm{A}_{2}$ and disk is 7.3 and $6.8 \mathrm{~mm}$, respectively. The distance between the aperture $A_{1}$ and the lens $\mathrm{L}_{1}$ is about $0.62 \mathrm{~m}$. The aperture $\mathrm{A}_{2}$ is far from the focal plane $0.58 \mathrm{~m}$ and the distance between the disk and the aperture $\mathrm{A}_{2}$ is about $0.25 \mathrm{~m}$. The laser pulses adjusted by an attenuator are separated into two beams by a splitter. The pulse energy of two beams is measured simultaneously by two energy detectors (Rjp-765 energy probe) linked to energy meter (Rj-7620 ENERGY RATIOMETER, Laserprobe). A personal computer is used to collect data coming from energy meter through GPIB interface.

Figure 4 shows the experiment results for the glass NAP2 using top-hat Z-scan and the modified Z-scan technique under the same experimental conditions, respectively. The nonlinear phase shift of the sample is so small that the signal cannot be detected using top-hat $\mathrm{Z}$-scan technique. However, the change of the normalized transmittance can be about 3.5 using our modified Z-scan technique. By varying the value of $\gamma$ to obtain the best fitting between the numerical calculated nonlinear transmittance and the experimental nonlinear transmittance, the value of $\gamma$ for NAP2 is obtained to be $n_{2}=8 \times 10^{-20} \mathrm{~m}^{2} / \mathrm{W}$.

\section{Conclusion}

In summary, we have reported a new method to characterize the nonlinear refraction index with high sensitivity. A single-shot technique based on the modified top-hat $\mathrm{Z}$-scan technique is also described. The modified technique provides a high-sensitivity method for measuring the nonlinear refraction of the materials which have small nonlinear phase shifts and can be damaged easily, such as nano-sized ultrathin film.

Acknowledgments We gratefully acknowledge the National Natural Science Fund of China (Grant No. 90922007), a project funded by the Priority Academic Program Development of Jiangsu Higher Education Institutions (PAPD), project supported by the Natural Science Foundation of Jiangsu Province, China (Grant No. BK2012178) and Research Fund for the Doctoral Program of Higher Education of China (RFDP, NO. 20113201120003).

Open Access This article is distributed under the terms of the Creative Commons Attribution License which permits any use, distribution, and reproduction in any medium, provided the original author(s) and the source are credited.

\section{References}

1. M. Sheik-Bahae, A.A. Said, E.W. Van Stryland, Opt. Lett. 14, 955 (1989)

2. M. Sheik-Bahae, A.A. Said, T.H. Wei, D.J. Hagan, E.W. Van Stryland, IEEE J. Quantum Electron. 26, 760 (1990)

3. S. Hughes, J.M. Burzler, Phys. Rev. A 56, R1103 (1995)

4. W. Zhao, P. Palffy-Muhoray, Appl. Phys. Lett. 63, 1613 (1993)

5. B. Gu, X.C. Peng, T. Jia, J.P. Ding, J.L. He, H.T. Wang, J. Opt. Soc. Am. B 22, 446 (2005)

6. T. Xia, D.J. Hagan, M. Sheik-Bahae, E.W. Van Stryland, Opt. Lett. 19, 317 (1994)

7. Z.B. Liu, S. Shi, X.Q. Yan, W.Y. Zhou, J.G. Tian, Opt. Lett. 36, $2086(2011)$

8. R. De Saint Denis, M. Fromager, E. Cagniot, K. Ait-Ameur, Appl. Phys. B 90, 513 (2008)

9. T. Godin, M. Fromager, E. Cagniot, R. Moncorgé, K. Aït-Ameur, Opt. Lett. 36, 1401 (1989)

10. G. Boudebs, S. Cherukulappurath, Phys. Rev. A 69, 053813 (2004)

11. S. Cherukulappurath, G. Boudebs, A. Monteil, J. Opt. Soc. Am. B 21, 273 (2004)

12. J.J. Hu, L. Zhang, W. Chen, C.H. Zhou, L.L. Hu, Chin. Opt. Lett. 10, 041403 (2012) 